\title{
The physical model for an organization: Similar aspects between "atom" and "organization"
}

\author{
Amir Pishkoo ${ }^{12}$ \\ ${ }^{1}$ Physics and Accelerators Research School, NSTRI, P.O. Box 14395-836, Tehran, Iran. \\ apishkoo@gmail.com
}

\begin{abstract} restructuring with nuclear reaction.

\section{Indexing terms/Keywords}

Physical system; Social system; Atom; Organization.

\section{Academic Discipline And Sub-Disciplines}

In this paper we claim that there exist some similar aspects between "atom", as a typical physical system, and its counterpart "organization" as a social system. These similar aspects have been shown by comparison between employees with electrons, board of directors with nucleus, dismissal with photoelectric effect, and organizational

Physics, Management, Atomic physics, Nuclear physics.

\section{INTRODUCTION}

Using the tools and methods existing in physics, human society can be analyzed by breaking it down into its components. If we first understand individual function of each component and their interaction together, society can be understood by generating the whole [1]. Many social phenomena such as cooperation, traffic dynamics, pedestrian movement [1-4] etc. have been equipped by mathematical descriptions that invoke self-organization. For instance Helbing et al. [5] develop the model for pedestrian motion which is able to reproduce many of the observed large-scale spatial features of trail systems. In [6] Helbing considers the empirical data and reviews the main approaches to modeling pedestrian and vehicle traffic. Pedestrian dynamics shows various collective phenomena such as lane formation and oscillatory flows through bottlenecks. According to the model proposed by Helbing et al. [7], the collective patterns of motion can be interpreted as self-organization phenomena which arise from the nonlinear interactions among pedestrians. In this paper we focus on organization, not society.

Society is spontaneous creation while an organization is intentional. Organization has clear purpose called mission statement and vision of its future. Furthermore, size of an organization can be clearly determined by number of members, or employees while it is more difficult to precisely determine the size of society. In order to compare organization with atom we should first use the term system for both; social system for an organization and physical system for an atom. A system is the fundamental concept which has been applied in many branches of sciences such as economic system in economics, chemical system in chemistry, physical system in physics, social system in social sciences [1,8-10] etc. It is formed of a set of interacting component parts which forms a whole. Focusing on just physical and social systems, a physical system is a portion of the physical universe chosen for analysis while a social system is the patterned series of interrelationships existing between individuals, groups, and institutions and forming a whole. In this manuscript we compare "organization" and "atom" in some aspects which shows their similarities. Knowing that they differ from some other aspects, we do not discuss to dissimilarities between them.

\section{ORGANIZATION AND ATOM}

An "organization" and "atom" as two fundamental concepts have several things in common. Organization is a social unit of people that is structured and managed to meet a need or to pursue collective goals. All organizations have a management structure that determines relationships between the different activities and the members, and subdivides and assigns roles, responsibilities, and authority to carry out different tasks. There exist an organization chart, a board of directors, an employer and employees who are arranged or rearranged based on processes like promotion, demotion, pension, recruitment, termination and organizational restructuring, etc. Organizations are open systems meaning that they affect and are affected by their environment.

On the other hand, an atom is a physical unit of matter which is the smallest unit of a chemical element. Each atom consists of a nucleus, which has a positive charge, and set of electrons that move around the nucleus. There exist energy levels, various atoms, nucleus, electrons which are arranged or rearranged based on processes like photoelectric effect, nuclear reaction, field electron emission, thermionic emission, etc. Now, we like to answer the following question:

What do a physical system like an atom, and a social system like an organization (see Fig. 1) has in common? To answer this question we claim that each of the following items should be compared together: 
- Employees \& electrons

- Board of directors \& nucleus

- Dismissal \& photoelectric effect

- Promotion \& electron transition from high energy level to low energy level

- Demotion \& electron transition from low energy level to high energy level

- Organizational restructuring \& nuclear reaction


Figure 1: Comparison of organization and atom.

\section{EMPLOYEES AND ELECTRONS}

An employee is anyone who has agreed to be employed, under a contract of service, to work for some form of payment. This includes wages, salary, commission and piece rates.

Electrons play a major role in all chemical bonds. They obey the Pauli exclusion principle which determines their positions in various energy levels, while employees are permission-based employment in various organizational positions. There exist free or bound electrons in metals. In much the same way, there are unemployed and employed people in society. The outermost electrons in the atoms are so loosely bound that they move in the space between the atoms of a conductor. Similarly, day laborers work temporarily for an organization.

\section{A BOARD OF DIRECTORS AND NUCLEUS}

A board of directors is a body of elected or appointed members who jointly oversee the activities of a company or organization, which can include a non-profit organization or a government agency or corporation.

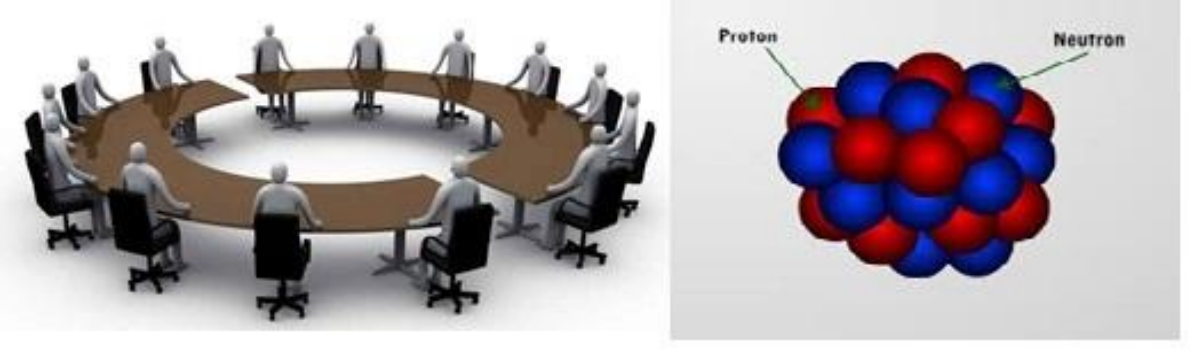

Figure 2: Comparison of board of directors with nucleus.

The nucleus has most of the mass of an atom, though it is only a very small part of it. Almost all of the mass in an atom is made up from the protons and neutrons in the nucleus with a very small contribution from the orbiting electrons. Neutrons have no charge and protons are positively charged.

There are power relations between boards and senior managers in organizations. In the same way, there exist strong bonds between protons and neutrons in the nucleus (see Fig. 2). Board members may have political (or economic) posturing such that they persist in their comments in the meetings. On the other hand, there are also some members that are neutral and conservative in all meetings. This board member is always listener in the meetings so that one just obeys or confirms others comments. Low-energy neutrons can easily penetrate inside the material while high-energy protons even may not penetrate within matter. Similarly, neutral and conservative persons have more penetrating instrument than factional persons.

\section{DISMISSAL AND PHOTOELECTRIC EFFECT}

Dismissal can be due to issues with the employee's performance, but it also may be due to factors outside the employee's control, such as downsizing, company restructuring, or the elimination of a position. Employers have a basic right to terminate the employment of an employee, but along with that right come responsibilities. 

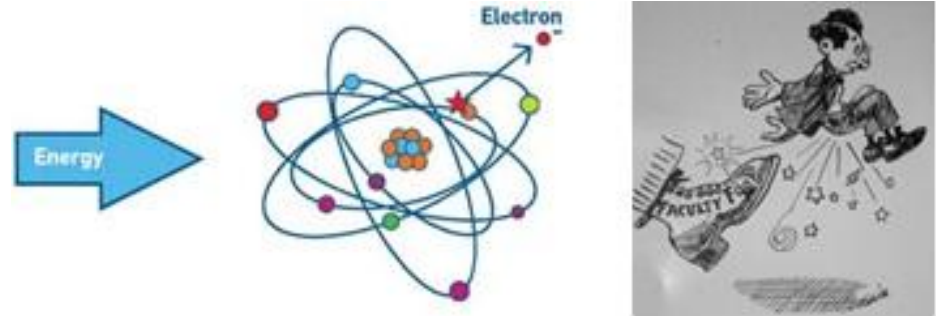

Figure 3: Comparison of Dismissal with Photoelectric effect.

The photoelectric effect refers to the emission, or ejection, of electrons from the surface of, generally, a metal in response to incident light. Energy contained within the incident light is absorbed by electrons within the metal, giving the electrons sufficient energy to be 'knocked' out of, that is, emitted from, the surface of the metal.

If there has been serious misconduct the employee can be dismissed without receiving any earlier notice or warning. Similarly, when light above a certain frequency (the threshold frequency) is shone on metals, this causes electrons to escape from the surface of metal (see Fig. 3). The escaping electrons are called photoelectrons. Presently, we can't distinguish retirement, redemption, and dismissal from each other. For all three the photo electric effect is just as counterpart.

\section{PROMOTION, DEMOTION AND ENERGY LEVELS}

A promotion is the advancement of an employee's rank or position in an organizational hierarchy system. Promotion may be an employee's reward for good performance, i.e., positive appraisal. Before a company promotes an employee to a particular position it ensures that the person is able to handle the added responsibilities by screening the employee with interviews and tests and giving them training or on-the-job experience. A promotion can involve advancement in terms of designation, salary and benefits, and in some organizations the type of job activities may change a great deal.
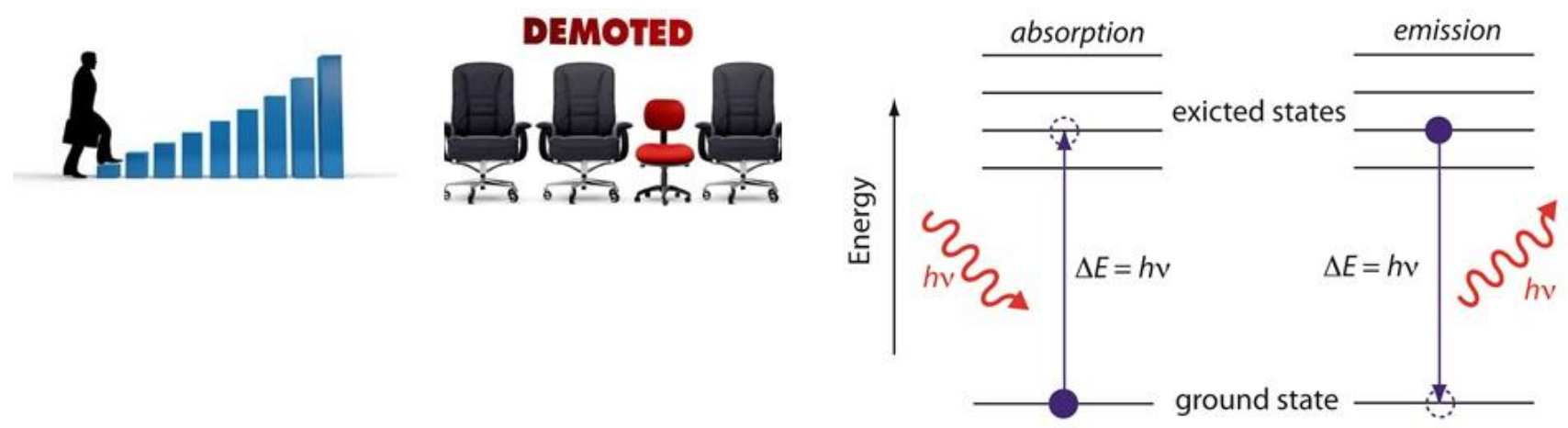

Figure 4: Comparison of Promotion, Demotion with Emission, Absorption.

A demotion is a compulsory reduction in an employee's rank or job title within the organizational hierarchy of a company, public service department, or other body. A demotion may also lead to the loss of other privileges associated with a more senior rank and/or a reduction in salary or benefits.

Energy levels inside an atom are the specific energies that electrons can have when occupying specific orbitals. Electrons can be excited to higher energy levels by absorbing energy from the surroundings. Light is emitted when an electron relaxes from a high energy state to a lower one (see Fig. 4). The ground state electron is closer to the nucleus than outer state (first, second, etc.) electrons. Promoted employee get more close to board of directors than demoted one.

\section{ORGANIZATIONAL RESTRUCTURING AND NUCLEAR REACTION}

Organizational structure determines how the roles, power and responsibilities are assigned, controlled, and coordinated, and how information flows between the different levels of management. A structure depends on the organization's objectives and strategy. An organizational chart illustrates the organizational structure. Organizational restructuring is the process by which an organization changes its internal structure by revamping departments, ownership, or operations and processes.

We may compare the organizational structure with energy levels in atom. In nuclear physics, a nuclear reaction is semantically considered to be the process in which two nuclei, or else a nucleus of an atom and a subatomic particle (such as a proton, neutron, or high energy electron) from outside the atom, collide to produce one or more nuclides that are different from the nuclide(s) that began the process. Thus, a nuclear reaction must cause a transformation of at least one nuclide to another. Similarly, it's never easy to split one company into two, but for one large communications company it was a challenge well worth attempting. When company leaders recognized that one of their profitable divisions should 
stand on its own as a separate company to help it grow and gain a competitive advantage, the rush was on to make it happen.

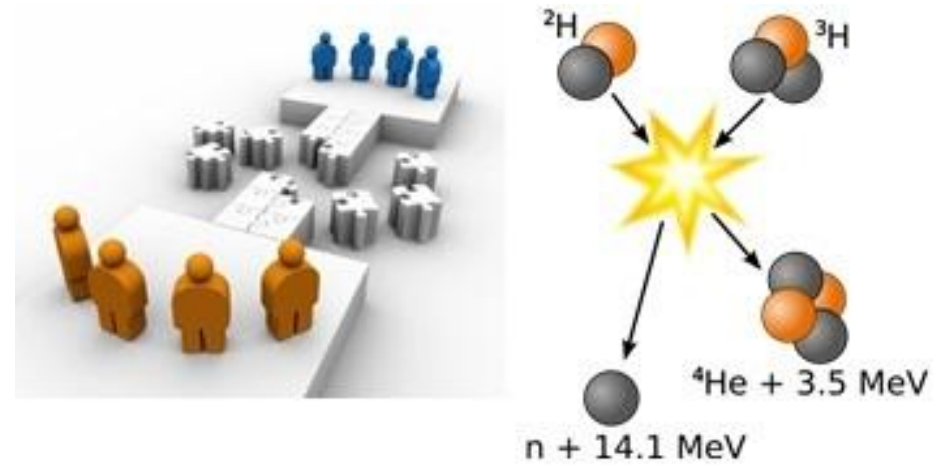

Figure 5: Comparison of Organizational Restructuring with Nuclear reaction.

In nuclear physics, nuclear fusion is a nuclear reaction in which two or more atomic nuclei come close enough to form one or more different atomic nuclei and subatomic particles (neutrons and/or protons). The difference in mass between the products and reactants is manifested as the release of large amounts of energy. In the same way, Consolidation occurs when two organizations or companies combine to form a new enterprise together, and neither of the previous organizations remains independently.

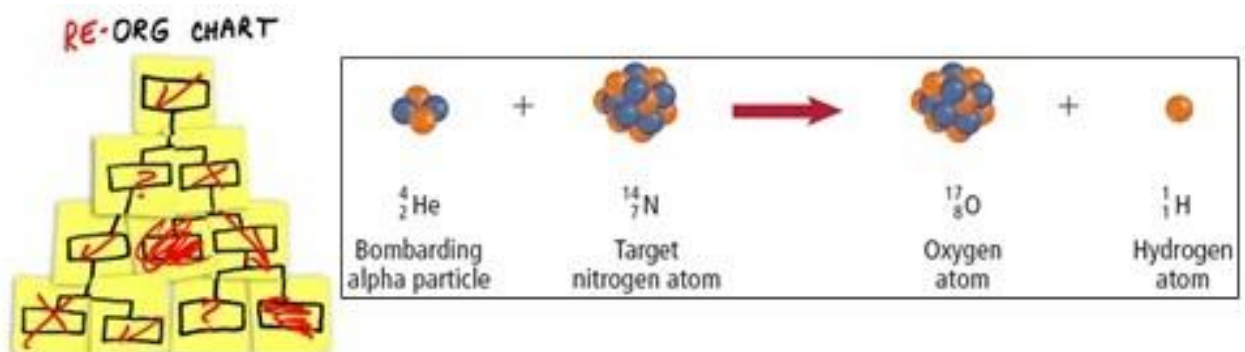

Figure 6: Comparison of Organizational Restructuring with Nuclear reaction2.

We can summarize our discussion with the key words of the two different sciences in the following table. This table can be made more complete in future by consideration and comparison more phenomena and processes in both social and physical sciences.

Table 1: Analyzed components of an typical social and physical system

\begin{tabular}{|c|c|}
\hline Social system & Physical system \\
\hline Organization & Atom \\
\hline Employees & Electrons \\
\hline Board of directors & Nucleus \\
\hline Dismissal & Photoelectric effect \\
\hline Promotion & Emission \\
\hline Demotion & Absorption \\
\hline Organizational structuring & Nuclear reaction \\
\hline
\end{tabular}

\section{CONCLUSION}

Although there is a fundamental difference between social laws and the laws of physics, this paper clarifies some similar aspects existing in "organization" and "atom" as typical examples of a social system and a physical system, respectively. So, it is likely that the study on the one physical phenomenon may lead to predicting a social event and vice versa. 


\section{REFERENCES}

[1] Ball, P. The physical modeling of human social systems. Complexus 2014; 1(4):190--206.

[2] Helbing, D., Keltsch, J., Molnar, P., Modelling the evolution of human trail systems. Nature, 1997;388:47--50.

[3] Jiang, R., Wu, Q-S., Zhu, Z-J., A new continuum model for traffic flow and numerical tests. Transportation Research Part B: Methodological, 2002; 36(5):405--419.

[4] Ross, P., Traffic dynamics. Transportation Research Part B: Methodological, 1988;22(6):421--435.

[5] Phase transitions in models of human cooperation. Physics Letters A, 2016; 380(36):2803--2808.

[6] Helbing, D. Traffic and related self-driven many-particle systems. Rev. Mod. Phys. 2001; 73(4):1067--1141.

[7] Helbing, D., Molnar, p., Farkas, I-J. Bolay, K., Self-organizing pedestrian movement. Environment and Planning B: Planning and Design, 2001; 28:361--383.

[8] Doyle, E., The economic system, Wiley; 2005.

[9] Bard, A-J., Integrated chemical systems: A chemical approach to nanotechnology, Jon Wiley \& Sons, Inc.; 1994.

[10] Parsons, T., The social system. Glencoe,III. : Free Press;1951. 